\title{
THE DIMENSION OF SOLUTION SETS TO SYSTEMS OF EQUATIONS IN ALGEBRAIC GROUPS
}

\author{
Anton A. Klyachko Maria A. Ryabtseva \\ Faculty of Mechanics and Mathematics of Moscow State University \\ Moscow 119991, Leninskie gory, MSU \\ klyachko@mech.math.msu.su ryabtseva.mariya@gmail.com
}

\begin{abstract}
The Gordon-Rodriguez-Villegas theorem says that, in a finite group, the number of solutions to a system of coefficientfree equations is divisible by the order of the group if the rank of the matrix composed of the exponent sums of $j$-th unknown in $i$-th equation is less than the number unknowns. We obtain analogues of this and similar facts for algebraic groups. In particular, our results imply that the dimension of each irreducible component of the variety of homomorphisms from a finitely generated group with infinite abelianisation into an algebraic group $G$ is at least $\operatorname{dim} G$.
\end{abstract}

\section{Introduction}

Solomon's theorem [Solo69]. In any group, the number of solutions to a system of coefficient-free equations is divisible by the order of the group if the system has less equations than unknowns.

Here as usual, an equation over a group $G$ is an expression of the form $v\left(x_{1}, \ldots, x_{m}\right)=1$, where $v$ is a word whose letters are unknowns, their inverses, and elements of $G$ called coefficients (there are no coefficients though by the condition of Solomon's theorem). In other words, the left-hand side of an equation is an element of the free product $G * F\left(x_{1}, \ldots, x_{m}\right)$ of $G$ and the free group $F\left(x_{1}, \ldots, x_{m}\right)$ of rank $m$ (where $m$ is the number of unknowns).

Solomon's theorem was generalised in different directions, see [Isaa70], [Stru95], [AmV11], [GRV12], [KM14], [KM17], [BKV18], and literature cited therein. For example, the following fact was proved in [KM14].

Theorem KM [KM14]. The number of solutions to a system of equations over a group is divisible by the order of the centraliser of the set of coefficients if the rank of the matrix composed of the exponent sums of the $j$-th unknown in the $i$-th equation is less than the number unknowns.

For coefficient-free equations, this theorem was obtained earlier by Gordon and Rodriguez-Villegas [GRV12] (and, in this case, the number of solutions is divisible by the order of the whole group). For example, the number of solutions to the system of equations $\left\{x^{100} y^{100}[x, y]^{777}=1,(x y)^{2020}=1\right\}$ is always divisible by the order of the group because this system (although not covered by Solomon's theorem) satisfies the conditions of the Gordon-RodriguezVillegas theorem: the matrix composed of the exponent sums (called the matrix of the system of equations) has the form $\left(\begin{array}{cc}100100 \\ 20202020\end{array}\right)$, and its rank is one (while the number of unknowns is two).

Note that, in these divisibility theorems, the group is not assumed to be necessarily finite; the divisibility is always understood in the sense of cardinal arithmetic: each infinite cardinal is divisible by all smaller nonzero cardinals. However, the most interesting applications of these theorems concern finite groups.

The purpose of this paper is to obtain analogues of the divisibility theorems for equations over algebraic groups. An analogue of the number of solutions to a system of equations is, in this case, the dimension of the variety of solutions. Slightly more precisely one can say that the dimension is something like the logarithm of the number of solutions. Note that the solutions of a (finite or infinite) system of equations in $m$ unknowns over an affine algebraic group $G$ form an affine algebraic subvariety in $G^{m}$. An analogue of the Solomon theorem is the following simple observation.

Theorem 0. In any affine algebraic group $G$, the dimension of each irreducible component of the variety of solutions to a finite system of equations (possibly with coefficients) is at least

$$
((\text { the number of unknowns })-(\text { the number of equations })) \cdot \operatorname{dim} G \text {. }
$$

Proof. The left-hand sides of $n$ equations in $m$ unknowns specify a morphism of algebraic varieties $\gamma: G^{m} \rightarrow G^{n}$. The solution variety is the fiber $\gamma^{-1}(1, \ldots, 1)$. Each irreducible component $M$ of the variety $G^{m}$ is isomorphic to $G_{0}^{m}$ as an algebraic variety (where $G_{0}$ is the identity component of $G$ ) and, therefore, $\operatorname{dim} M=m \cdot \operatorname{dim} G$. The restriction of $\gamma$ to $M$ is a dominant morphism of irreducible algebraic varieties $M \rightarrow N$, where $N$ is the Zariski closure of $\gamma(M)$ (this morphism is dominant because any set is dense in its closure). It remains to refer to the following general fact (see, e.g., [Bo72]).

The work of the first author was supported by the Russian Foundation for Basic Research, project no. 19-01-00591. 
Fiber lemma. If $\gamma: M \rightarrow N$ is a dominant morphism of irreducible algebraic varieties, then, for any point $s \in f(M)$, the dimension of the fiber $\gamma^{-1}(s)$ is at least $\operatorname{dim} M-\operatorname{dim} N$; moreover, $N$ contains a nonempty open subset $U \subset f(X)$ such that $\operatorname{dim} \gamma^{-1}(u)=\operatorname{dim} M-\operatorname{dim} N$ for any $u \in U$.

Theorem 0 is hardly new; for coefficient-free equations, this fact was noted, e.g., in [LM11].

Solomon's theorem looks better than Theorem 0 in the sense that Solomon's theorem asserts divisibility, while Theorem 0 only asserts an inequality (this is unavailable - the divisibility of dimensions would be too much of course). In all other aspects, Theorem 0 looks better; but one cannot improve Solomon's theorem accordingly as the following simple examples show.

False theorem. In any group $G$, the number of solutions to a consistent system of equations with more unknowns than equations

1) is at least the order of the group;

2 ) is a multiple of $|G|^{\text {(the number of unknowns)-(the number of equations) }}$ if the system is coefficient-free.

\section{Proof.}

1) The first assertion is false for a simple reason: in the symmetric group of order six, the equation $x^{3} y^{3}=\left(\begin{array}{lll}1 & 2 & 3\end{array}\right)$ has precisely three solutions, because $x$ and $y$ must have the same parity. Moreover, they cannot be even, because the cube of an even permutation is identity. The product of two different transpositions is always a 3-cycle; three of these six products give one 3-cycle, and three give the other. (Surely, the number of solutions must be a multiple of three by Theorem KM.)

2) The second assertion is also false as the equation $x^{2} y^{2} z^{2}=1$ in the same symmetric group shows. The squares of permutations are even; therefore, for these squares, we have three possibilities:

- they are the same 3-cycle;

- or one of the squares is the identity permutation, and the other two are different 3-cycles;

- or all three square are the identity permutation.

The identity permutation has four square roots, a 3 -cycle has one square root, and we obtain $2+3 \cdot 2 \cdot 4+4 \cdot 4 \cdot 4=90$ solutions.

The following fact is an analogue of Theorem KM.

Theorem 1. If the rank of the matrix of a system of equations with finitely many unknowns over an affine algebraic group is less than the number of unknowns, then the dimension of each component of the variety of solutions to this system is at least the dimension of the centraliser of the set of coefficients.

The equation $[x, y]=1$ (with zero matrix and two unknowns) over a nonabelian connected group shows that the estimate from Theorem 1 (even for coefficient-free case) cannot be strengthened to the inequality

$\operatorname{dim}($ the variety of solutions $) \geqslant(($ the number of unknowns $)-($ rank of the matrix of the system $)) \cdot \operatorname{dim} G$ (similar to Theorem 0).

Question (suggested by the referee). Is it true that, under the conditions of Theorem 1, the class of the solution variety is a multiple of the class of the centraliser of the coefficients in the Grothendieck ring of varieties?

If yes, then it would include both Theorem 1 and Theorem KM (for finite groups).

Corollary (or rather a reformulation of Theorem 1 for coefficient-free equations). Suppose that $G$ is an affine algebraic group and $F$ is a finitely generated group whose commutator subgroup is of infinite index. Then the dimension of each irreducible component of the variety of homomorphisms $F \rightarrow G$ is at least $\operatorname{dim} G$.

The representation variety (or homomorphism variety) $\operatorname{Hom}(F, G)$ of a finitely generated group $F$ in an algebraic group $G$ was studied in many papers, see, e.g., [RBCh96], [MO10], [LM11], [LL13], [LS05], [Ki18], [LT18] and literature cited therein. In particular, it was studied when there exist injective or topologically surjective homomorphisms, i.e. homomorphisms with a dense (in the Zariski topology) image, and how many such homomorphisms are there. For example, in [Ki18] it was shown that topologically surjective homomorphisms from the fundamental group of a closed oriented surface of genus larger than one into a real semisimple algebraic group always exist, and there are a lot of them in some sense. In [BGGT12], it was shown that there exists a homomorphism from any free group into almost any semisimple algebraic group such that the restriction of the homomorphism to any noncyclic subgroup is topologically surjective. See also a recent survey [GKP18].

Generally, neither injective nor topologically surjective homomorphisms form a variety; however, the following theorem shows that, in some sense, all "components" of these (and similar) non-varieties have dimension not less than that of the commutator subgroup of $G$. We consider the following property of a homomorphism $\varphi: F \rightarrow G$ from a finitely generated group $F$ containing a subgroup $W$ into an algebraic group $G$ containing a closed subgroup $A$.

$\mathbf{F}_{W, A}$ (faithfulness): $\varphi(W) \subseteq A$ and the restriction of $\varphi$ to $W \subseteq F$ is injective;

$\mathbf{S}_{W, A}$ (topological surjectivity): the closure of the subgroup $\varphi(W)$ is $A$;

$\mathbf{C}_{W, A}$ (connectivity): $\varphi(W) \subseteq A$ and the closure of $\varphi(W)$ is irreducible. 
Theorem 2. Suppose that $A$ is a subgroup of an affine algebraic group $G$, and $F$ is a finitely generated group whose commutator subgroup is of infinite index. Then each homomorphism $\alpha: F \rightarrow G$ having some (possibly infinite) combination (conjunction)

$$
\mathbf{P}=\left(\bigwedge_{W \in \mathcal{F}} \mathbf{F}_{W, A}\right) \wedge\left(\bigwedge_{W \in \mathcal{S}} \mathbf{S}_{W, A}\right) \wedge\left(\bigwedge_{W \in \mathcal{C}} \mathbf{C}_{W, A}\right), \quad \text { where } \mathcal{F}, \mathcal{S}, \mathcal{C} \text { are some family of subgroups of } F,
$$

of the properties listed above is contained in a $(\operatorname{dim}[A, A])$-dimensional irreducible subvariety of $\operatorname{Hom}(F, G)$ consisting of homomorphisms having the same combination of properties $\mathbf{P}$ provided the index of the subgroup $[F, F] \cdot \prod_{W \in \mathcal{F} \cup \mathcal{C}} W$

in $F$ is infinite (and no conditions on the family $\mathcal{S}$ are imposed).

Here, one cannot replace the dimension of the commutator subgroup with the dimension of the entire group as the following simple example shows: there are many topologically surjective homomorphisms from the infinite cyclic group to the torus $\left(\mathbb{C}^{*}\right)^{2020}$ but they are all lone in the sense that any non-zero-dimensional subvariety of Hom $\left(\mathbb{Z},\left(\mathbb{C}^{*}\right)^{2020}\right)$ contains a homomorphism that is not topologically surjective (because any infinite subvariety in $\mathbb{C}^{*}$ contains a finiteorder element).

One cannot also remove the condition on the family $\mathcal{F}$ and $\mathcal{C}$ as simple examples of homomorphisms from $\mathbb{Z}$ to $\mathbf{S L}_{n}(\mathbb{C})$ show.

For instance, Theorem 2 implies that

if a homomorphism from the fundamental group $F=\langle x, y, z, t \mid[x, y]=[z, t]\rangle$ of the genus-two orientable surface (or from any other group with more generators than relators) into an algebraic group $G$ is injective on the subgroup $\langle x, y, z\rangle$ and maps any non-abelian subgroup onto a subgroup dense in $G$, then it is contained in a $(\operatorname{dim}[G, G])$-dimensional subvariety of $\operatorname{Hom}(F, G)$ consisting of homomorphisms with the same two properties.

Partially, Theorem 2 is an analogue of the following known fact [KM17]:

the number of surjective homomorphisms from a finitely generated group whose commutator subgroup is of infinite index into a (finite if you like) group $G$ is divisible by the order of the commutator subgroup of $G$. For example, the number of pairs of elements generating a (2-generated) group is always divisible by the order of the commutator subgroup of this group.

However, in "algebraic settings" some new effects arise.

Theorems 1 and 2 are special cases of a more general Main theorem, which is stated in the next section. Our main theorem is a direct "algebraic" analogue of the main theorem from [KM17] (whose special cases are all theorems on divisibility mentioned above and some other curious facts, see [KM17] and [BKV18]).

The authors thank D. A. Timashev and an anonymous referee for valuable remarks.

Our Notation and conventions are mainly standard. Note only that, if $k \in \mathbb{Z}$, and $x$ and $y$ are elements some group, then $x^{y}, x^{k y}$, and $x^{-y}$ denote $y^{-1} x y, y^{-1} x^{k} y$, and $y^{-1} x^{-1} y$, respectively. The commutator subgroup of a group $G$ is denoted by $G^{\prime}$ or $[G, G]$. If $X$ is a subset of a group, then $\langle X\rangle,\langle\langle X\rangle$, and $C(X)$ mean the subgroup generated by $X$, normal closure of $X$, and the centraliser of $X$, respectively, The index of a subgroup $H$ of a group $G$ is denoted $|G: H|$. The letter $\mathbb{Z}$ denotes the set of integers. The symbol $\operatorname{Hom}(A, B)$ denotes the set of homomorphisms from a group $A$ to a group $B$. The free group of rank $n$ is denoted as $F\left(x_{1}, \ldots, x_{n}\right)$ or $F_{n}$. The symbol $A * B$ denotes the free product of groups $A$ and $B$. The word variety always means a (not necessarily irreducible) quasi-projective algebraic variety over an algebraically closed field (of arbitrary characteristic); the word subvariety means a locally closed subset of a variety. All topological terms refer to the Zariski topology.

\section{Main theorem}

A group $F$ equipped with an epimorphism $F \rightarrow \mathbb{Z}$ is called an indexed group. This epimorphism $F \rightarrow \mathbb{Z}$ is called the degree or indexing and denoted as deg; thus, for any element $f$ of an indexed group $F$, there is an integer $\operatorname{deg} f$, the group $F$ contains elements of all integer degrees, and $\operatorname{deg}(f g)=\operatorname{deg} f+\operatorname{deg} g$ for any $f, g \in F$.

Suppose that $\varphi: F \rightarrow G$ is a homomorphism from an indexed group $F$ to some group $G$, and $H$ is a subgroup of $G$. We call the subgroup

$$
H_{\varphi}=\bigcap_{f \in F} H^{\varphi(f)} \cap C(\{\varphi(f) \mid \operatorname{deg} f=0\})
$$

the $\varphi$-core of $H$. In other words, the $\varphi$-core $H_{\varphi}$ of $H$ consists of its elements $h$ such that $h^{\varphi(f)} \in H$ for all $f$ and $h^{\varphi(f)}=h$ if $\operatorname{deg} f=0$. 
Main theorem. Suppose that $H$ is an affine algebraic subgroup of an algebraic group $G$, and a subvariety $\Phi$ of $\operatorname{Hom}(F, G)$, where $F$ is a finitely generated indexed group, has the following two properties.

I. $\Phi$ is invariant under conjugation by elements of $H$ :

$$
\text { if } h \in H \text { and } \varphi \in \Phi \text {, then the homomorphism } \psi: f \mapsto \varphi(f)^{h} \text { also lies in } \Phi \text {. }
$$

II. For any $\varphi \in \Phi$ and any element $h$ of the $\varphi$-core $H_{\varphi}$ of $H$, the homomorphism $\psi$ defined as

$$
\psi(f)= \begin{cases}\varphi(f) & \text { for all elements } f \in F \text { of degree zero; } \\ \varphi(f) h & \text { for some element } f \in F \text { of degree one (and, hence, for all elements of degree one) }\end{cases}
$$

belongs to $\Phi$ too.

Then the dimension of each irreducible component of $\Phi$ is at least the dimension of $H$.

Note that the mapping $\psi$ from Condition I is a homomorphism for any $h \in G$, and the formula for $\psi$ from condition II defines a homomorphism for any $h \in C(\varphi(\operatorname{ker} \operatorname{deg}))$ ([KM17], Lemma 0$)$. Conditions I and II only require these homomorphisms to belong to $\Phi$ (under some additional restrictions on $h$ ).

This theorem is an analogue of the main theorem in [KM17], which asserts that, for any (abstract) group $G$ and any its subgroup $H$, the cardinality of a set $\Phi$ of homomorphisms from an indexed group $F$ to $G$ is divisible by $|H|$ if Conditions I and II hold.

\section{Proof of Theorem 1}

Let $A \subseteq G$ be the subgroup generated by all coefficients of all equations, and let $F$ be the quotient group

$$
F=\left(A * F\left(x_{1}, \ldots, x_{n}\right)\right) /\left\langle\left\langle\left\{v_{i}\right\}\right\rangle\right\rangle
$$

of the free product $A * F\left(x_{1}, \ldots, x_{n}\right)$ of $A$ and the free group $F\left(x_{1}, \ldots, x_{n}\right)$ by the normal subgroup $\left\langle\left\langle\left\{v_{i}\right\}\right\rangle\right\rangle$ generated by the left-hand sides of all equations. As the set $\Phi$ we consider the set of homomorphisms $F \rightarrow G$ which restrict to the identity on $A$ (we assume that the natural mapping $A \rightarrow F$ is an embedding because otherwise equations have no solutions and we have nothing to prove). Clearly, the solutions to the system of equations are in a natural one-to-one correspondence with the elements of $\Phi$, and $\Phi$ is a subvariety of $\operatorname{Hom}(F, G)$.

The condition on the rank means that $F$ admits an epimorphism onto $\mathbb{Z}$ whose kernel contains $A$. Now, take the centraliser of $A$ in $G$ as $H$. The conditions of the main theorem are obviously fulfilled. Indeed, Condition I holds because $h$ centralises $A \subseteq G$ and, hence, $\psi$ coincides with $\varphi$ on $A \subset F$; Condition II holds because elements of $A \subset F$ have degree zero and, therefore, $\psi$ coincides with $\varphi$ on $A \subset F$ - again.

\section{Proof of Theorem 2}

If the commutator subgroup of a finitely generated group is of infinite index, then, as is known, the group admits an epimorphism onto $\mathbb{Z}$. Therefore, the conditions of Theorem 2 implies the existence of an indexing deg: $F \rightarrow \mathbb{Z}$ whose kernel contains all subgroup from the families $\mathcal{C}$ and $\mathcal{F}$ (and the commutator subgroup $F^{\prime}$ of course). Let $H$ be the commutator subgroup $A^{\prime}$ of $A$, and let $\Phi$ be the set of homomorphisms $F \rightarrow G$ coinciding with a given homomorphism $\alpha: F \rightarrow G$ modulo $H$ and coinciding with $\alpha$ on elements of degree zero:

$$
\Phi=\{\varphi: F \rightarrow G \mid \quad \varphi(f) H=\alpha(f) H \text { for all } f \in F ; \quad \varphi(f)=\alpha(f) \text { for all } f \in \operatorname{ker} \operatorname{deg}\} .
$$

Clearly, the conditions of the main theorem hold for these $F$, deg, $\Phi$, and $H$. Therefore, the dimension of each component of the variety $\Phi$ is at least $\operatorname{dim} H=\operatorname{dim} A^{\prime}$. It remains to verify that, if the homomorphism $\alpha$ has the property $\mathbf{P}$, then all homomorphisms from $\Phi$ have this property too. Suppose that $\varphi \in \Phi$, i.e. $\varphi(f)=\alpha(f) h_{f}$ for all $f \in F$, where $h_{f} \in H=A^{\prime}$.

First, note that the subgroup $\varphi\left(W^{\prime}\right)=\alpha\left(W^{\prime}\right)$ is dense in $H=A^{\prime}$ for all $W \in \mathcal{S}$. Indeed, any algebraic group has finite commutator width, i.e. the morphism $\varkappa: A^{2 n} \rightarrow A^{\prime}=H$ sending $\left(x_{1}, \ldots, x_{n}, y_{1}, \ldots, y_{n}\right)$ to the product of commutators $\prod\left[x_{i}, y_{i}\right]$ is surjective for sufficiently large $n$ (see, e.g., [VO88]). The image of a dense set under a continuous surjective mapping is dense (and $D^{2 n}$ is dense in $A^{2 n}$ if $D$ is dense in $A$ ). Thus, any nonempty open subset of $A^{\prime}=H$ contains an element of $\varkappa\left((\alpha(W))^{2 n}\right) \subseteq \alpha\left(W^{\prime}\right)=\varphi\left(W^{\prime}\right)$ as required.

Let $U \subseteq A$ be a nonempty open set. Since $\alpha(W)$ is dense in $A$ (where $W \in \mathcal{S}$ ), the set $U$ contains an element $\alpha(w)=\varphi(w) h_{w}^{-1}$, where $w \in W$. Therefore, the open set $\varphi\left(w^{-1}\right) U$ contains $h_{w}^{-1} \in H=A^{\prime}$. Hence, $\varphi\left(w^{-1}\right) U \cap A^{\prime}$ is a nonempty open subset in $A^{\prime}=H$. As shown above, it contains some element $\varphi\left(w_{1}\right)$, where $w_{1} \in W$. Therefore, $U \ni \varphi\left(w w_{1}\right)$, and this completes the proof of property $\mathbf{S}_{W, A}$ for $\varphi$.

Properties $\mathbf{F}_{W, A}$ (where $W \in \mathcal{F}$ ) and $\mathbf{C}_{W, A}$ (where $W \in \mathcal{C}$ ) hold for the obvious reason: all these subgroup $W$ consist of elements of degree zero (by the choice of deg), therefore, $\varphi$ and $\alpha$ coincide on all such subgroups $W$. 


\section{Proof of the main theorem}

We follow the proof of the main theorem in [KM17] with necessary modifications. The first difficulty is that the subgroup ker deg $\subset F$ may be non-finitely generated and, therefore, the set of homomorphisms ker deg $\rightarrow G$ may have no natural structure of an algebraic variety. To overcome this unpleasant feature we use the following simple observation:

there exists a finite subset $K \subseteq \operatorname{ker} \operatorname{deg} \subset F$ such that any two homomorphism from $F$ to $G$ coinciding on $K$ coincide on ker deg.

Indeed, suppose that kerdeg $=\left\{d_{1}, d_{2}, \ldots\right\}$ and let $\Pi_{i}$ be the set of pairs of homomorphisms $F \rightarrow G$ coinciding on $d_{1}, \ldots, d_{i}$. Clearly, $\Pi_{i}$ form a decreasing chain of subvarieties in $\operatorname{Hom}(F, G) \times \operatorname{Hom}(F, G)$. Such a chain must stabilise: $\Pi_{n}=\Pi_{n+1}=\ldots$ for some $n$. Therefore, we can put $K=\left\{d_{1}, d_{2}, \ldots, d_{n}\right\}$.

We also need a similar fact:

there exists a finite subset $A \subset F$ such that, if, for two homomorphisms $\alpha$ and $\beta$ from $F$ to $G$, their composition with the natural mapping $G \rightarrow G / H$ (where $G / H$ is the set of left cosets of $H$ in $G$ ) coincide on $A$, then they coincide on the entire group $F$ :

$$
\forall \alpha, \beta \in \operatorname{Hom}(F, G) \quad(\forall a \in A \quad \alpha(a) H=\beta(a) H) \Longrightarrow(\forall f \in F \quad \alpha(f) H=\beta(f) H) .
$$

Indeed, suppose now that $F=\left\{d_{1}, d_{2}, \ldots\right\}$ and $\Pi_{i}$ is the set of pairs of homomorphisms $\alpha, \beta: F \rightarrow G$ such that $\alpha\left(d_{k}\right) H=\beta\left(d_{k}\right) H$ for $k \leqslant i$. Clearly, $\Pi_{i}$ form a decreasing chain of subvarieties. Such a chain must stabilise: $\Pi_{n}=\Pi_{n+1}=\ldots$ for some $n$. Therefore, we can put $A=\left\{d_{1}, d_{2}, \ldots, d_{n}\right\}$.

Now, consider the variety $X=G^{K} \times(G / H)^{A}$ consisting of all pairs of mappings $K \rightarrow G$ and $A \rightarrow G / H$ (where $G / H$ is the variety of left cosets the subgroup $H$ in $G$ ). The group $H$ acts on $X$ (by conjugations):

$$
h \circ(\alpha, \beta)=\left(f \mapsto h \alpha(f) h^{-1}, a \mapsto h \beta(a)\right) .
$$

The tail $\chi(\varphi)$ of a homomorphism $\varphi: F \rightarrow G$ is the pair $\left(\varphi_{0}, \varphi_{H}\right)$, where $\varphi_{0}$ is restriction of $\varphi$ to the set $K \subseteq \operatorname{ker} \operatorname{deg} \subset F$, and $\varphi_{H}: A \rightarrow\{g H ; g \in G\}$ is the mapping from $A$ to $G / H$ which maps $a \in A$ to the coset $\varphi(a) H$. Clearly, the mapping $\chi: \operatorname{Hom}(F, G) \rightarrow X$ is a morphism of algebraic varieties.

We say that two homomorphism $\varphi, \psi \in \Phi$ are similar and write $\varphi \sim \psi$ if their tails lie in the same orbit under the action of $H$ on $X$ described above. Note that neither the similarity of homomorphisms nor coincidence of their tails depend on the choice of sets $A$ and $K$ (these sets are needed solely to make "taking the tail" a morphism of algebraic varieties and to make the action of $H$ on tails an action of an algebraic group on an algebraic variety):

$$
\begin{gathered}
\chi(\varphi)=\chi(\psi) \Longleftrightarrow \begin{cases}\psi(f)=\varphi(f) & \text { for all } f \in F \text { of degree zero and } \\
\psi(f) H=\varphi(f) H & \text { for all } f \in F .\end{cases} \\
\varphi \sim \psi \Longleftrightarrow \text { for some } h \in H \begin{cases}\psi(f)=h \varphi(f) h^{-1} & \text { for all } f \in F \text { of degree zero and } \\
\psi(f) H=h \varphi(f) H & \text { for all } f \in F .\end{cases}
\end{gathered}
$$

Without loss generality, we assume that the group $H$ is irreducible (because the identity component of $H$ is a group of the same dimension).

Each class of similar homomorphisms is a locally closed subvariety in $\operatorname{Hom}(F, G)$ because this class is the preimage of an orbit under the morphism $\chi$, and the orbit of an action of an algebraic group on an algebraic variety is always locally closed (see, e.g., [VO88]). The main theorem follows immediately from the following proposition.

Proposition. The dimension of each component of each class of similar homomorphisms in $\Phi$ is $\operatorname{dim} H$. More precisely, for each $\varphi \in \Phi$,

1) the dimension of the variety $X_{\varphi}$ of tails of homomorphisms from $\Phi$ similar to $\varphi$ equals $\operatorname{dim} H-\operatorname{dim} H_{\varphi}$;

2) for each homomorphism $\psi$ similar to $\varphi$, the dimension of each component of the variety of homomorphisms from $\Phi$ with the same tail as $\psi$ equals $\operatorname{dim} H_{\varphi}$.

Proof. To prove Assertion 1) note that the set $\chi(\Phi) \subseteq X$ is invariant with respect to the action of $H$ on $X$. Indeed, $h \circ \chi(\varphi)=\chi\left(f \mapsto h \varphi(f) h^{-1}\right)$. This homomorphism $f \mapsto h \varphi(f) h^{-1}$ lies in $\Phi$ by Condition I of the main theorem. The tails of homomorphisms similar to $\varphi$ is the orbit of the tail of $\varphi$ under this action. The dimension of an orbit is, as is known, equal to the codimension of the stabiliser (this is a special case of the fiber lemma). It remains to note that the subgroup $H_{\varphi}$ is the stabiliser of the tail of $\varphi$ (by formula $(*)$ ).

Let us prove the second assertion. Choose an element $x \in F$ of degree one. A homomorphism $\alpha: F \rightarrow G$ is uniquely determined by its tail and the value $\alpha(x)$ (by formula $(*)$ ). Moreover, for two homomorphisms $\alpha$ and $\beta$ with the same tail, the quotient $h=(\alpha(x))^{-1} \beta(x)$ must stabilise this tail, i.e. lie in $H_{\alpha}$. Indeed, for all $f \in F$ of degree zero we have

$$
\alpha\left(f^{x}\right)^{h}=\alpha(f)^{\alpha(x) h}=\alpha(f)^{\beta(x)} \stackrel{*}{=} \beta(f)^{\beta(x)}=\beta\left(f^{x}\right) \stackrel{*}{=} \alpha\left(f^{x}\right), \quad \text { i.e. } h \text { centralises the subgroup } \alpha(\text { ker deg })
$$


and, for any element $f \in F$, we have

$$
\alpha(x) \alpha(f) H=\alpha(x f) H \stackrel{*}{=} \beta(x f) H=\beta(x) \beta(f) H=\alpha(x) h \beta(f) H \stackrel{*}{=} \alpha(x) h \alpha(f) H, \quad \text { i.e. } h \in \alpha(f) H \alpha(f)^{-1} .
$$

(Here, equalities $\stackrel{*}{=}$ follow from formula $(*)$.) Thus, $h=(\alpha(x))^{-1} \beta(x) \in H_{\alpha}$.

On the other hand, if $h$ is an element of $H_{\alpha}$, then the mapping

$$
f \mapsto \begin{cases}\alpha(f), & \text { if } \operatorname{deg} f=0 \\ \alpha(x) h, & \text { if } f=x\end{cases}
$$

can obviously be extended to a homomorphism with the same tail as $\alpha$ (see the remark after the statement of the main theorem in Section 2). This homomorphism lies in $\Phi$ by Condition II of the main theorem.

We showed that, for any $\alpha \in \Phi$, the mapping $H_{\alpha} \rightarrow \operatorname{Hom}(F, G)$ that maps an element $h \in H_{\alpha}$ to the homomorphism $(* *)$ is an injective morphism of algebraic varieties whose image is the set of elements of $\Phi$ with the same tail as $\alpha$. This means that $\operatorname{dim} \chi^{-1}(\chi(\alpha))=\operatorname{dim} H_{\alpha}$.

It remains to note that, for similar homomorphisms $\psi$ and $\varphi$, the subgroup $H_{\varphi}$ and $H_{\psi}$ are isomorphic and even conjugate in $H$ because they are the stabilisers of points $\chi(\varphi)$ and $\chi(\psi)$ lying in the same orbit under the action of $H$ on $X$. This completes the proof of Assertion 2).

The variety $X_{\varphi}$ is irreducible (because we assume that the group $H$ is irreducible) and each component $\Pi_{\varphi} \ni \varphi$ of the variety of homomorphisms similar to $\varphi$ maps onto $X_{\varphi}$ surjectively because $\chi\left(f \mapsto h \varphi(f) h^{-1}\right)=h \circ \chi(\varphi)$ and the variety $\left\{f \mapsto h \varphi(f) h^{-1} \mid h \in H\right\}$ is irreducible (as it is an orbit under the action of the connected group $H$ on $\operatorname{Hom}(F, G)$ ). Therefore, 1$), 2$ ), and the following general fact imply that the dimension of each component of each class of similar homomorphisms from $\Phi$ is $\operatorname{dim} H$.

Lemma. Let $\gamma$ be a morphism from a variety $P$ to an irreducible variety $N$ such that all components of all fibers $\gamma^{-1}(y)$ (where $y \in N$ ) have the same dimension $d$ and the image of each component of $P$ is dense in $N$. Then the dimension of each component of $P$ is $\operatorname{dim} N+d$.

Proof. Let $M$ be an irreducible component of $P$. Take an open nonempty subset $U \subseteq \gamma(M) \subseteq N$ from the fiber lemma. Irreducibility of $M$ implies that $\gamma^{-1}(U)$ contains a point $x$ not belonging to other components of $P$. Thus, each component $K$ of the fiber $\gamma^{-1}(\gamma(x))$ containing $x$ must lie in $M$ and, therefore, be a component of the fiber of the restriction of the morphism $\gamma$ to $M$. Clearly, the other components of this fiber $M \cap \gamma^{-1}(\gamma(x))$ have dimension at most $d=\operatorname{dim} K$. By the fiber lemma, we obtain $\operatorname{dim} M=\operatorname{dim} K+\operatorname{dim} N$ as required. This completes the proofs of the lemma and main theorem.

\section{REFERENCES}

[Bo72] A. Borel, Linear algebraic groups. Benjamin, New York, 1969.

[VO88] E. B. Vinberg, A. L. Onishchik, Seminar on Lie groups and algebraic groups. Springer Verlag, 1990.

[GKP18] N. L. Gordeev, B. E. Kunyavskiŭ, E. B. Plotkin, Geometry of word equations in simple algebraic groups over special fields, Russian Mathematical Surveys, 73:5(443) (2018), 753-796. See also arXiv:1808.02303.

[Stru95] S. P. Strunkov, On the theory of equations in finite groups, Izvestiya: Mathematics, 59:6 (1995), 1273-1282.

[AmV11] A. Amit, U. Vishne, Characters and solutions to equations in finite groups, J. Algebra Its Appl., 10:4 (2011), 675-686.

[BGGT12] E. Breuillard, B.Green, R. Guralnick, and T. Tao, Strongly dense free subgroups of semisimple algebraic groups, Israel Journal of Mathematics, 192:1 (2012), 347-379. See also arXiv:1010.4259.

[BKV18] E. K. Brusyanskaya, A. A. Klyachko, A. V. Vasil'ev, What do Frobenius's, Solomon's, and Iwasaki's theorems on divisibility in groups have in common?, Pacific Journal of Mathematics, 302:2 (2019), 437-452. See also arXiv: 1806.08870 .

[GRV12] C. Gordon, F. Rodriguez-Villegas, On the divisibility of $\# \operatorname{Hom}(\Gamma, G)$ by $|G|$, J. Algebra, 350:1 (2012), 300-307. See also arXiv:1105.6066.

[Isaa70] I. M. Isaacs, Systems of equations and generalized characters in groups, Canad. J. Math., 22 (1970), 1040-1046.

[Ki18] K. Kishore, Representation variety of surface groups, Proc. Amer. Math. Soc. 146 (2018), 953-959. See also arXiv:1702.05981.

[KM14] A. A. Klyachko, A. A. Mkrtchyan, How many tuples of group elements have a given property? With an appendix by Dmitrii V. Trushin, Intern. J. of Algebra and Comp. 24:4 (2014), 413-428. arXiv 1205.2824

[KM17] A. A. Klyachko, A. A. Mkrtchyan, Strange divisibility in groups and rings, Arch. Math. 108:5 (2017), 441-451. See also arXiv:1506.08967.

[LL13] M. Larsen, A. Lubotzky, Representation varieties of Fuchsian groups, From Fourier analysis and number theory to radon transforms and geometry, 375-397, Dev. Math., 28, Springer, New York, 2013.

See also arXiv:1203.3408.

[LS05] M. Liebeck, A. Shalev, Fuchsian groups, finite simple groups and representation varieties, Inventiones mathematicae 159:2 (2005), 317-367. 
[LM11] S. Liriano, S. Majewicz, Algebro-geometric invariants of groups (the dimension sequence of representation variety), Int. J. Algebra Comput., 21:4 (2011), 595-614.

[LT18] D. D. Long, M. B. Thistlethwaite, The dimension of the Hitchin component for triangle groups, Geometriae Dedicata (to appear).

[MO10] J. Martín-Morales, A. M. Oller-Marcén, On the number of irreducible components of the representation variety of a family of one-relator groups, Internat. J. Algebra Comput. 20:1 (2010), 77-87. See also arXiv:0805.4716.

[RBCh96] A. S. Rapinchuk, V. V. Benyash-Krivetz, V. I. Chernousov, Representation varieties of the fundamental groups of compact orientable surfaces, Israel Journal of Mathematics, 93:1 (1996), 29-71.

[Solo69] L. Solomon, The solutions of equations in groups, Arch. Math., 20:3 (1969), 241-247. 